\title{
A combined approach of generalized additive model and bootstrap with small sample sets for fault diagnosis in fermentation process of glutamate
}

\author{
Chunbo Liu ${ }^{1,2^{*}}$, Feng Pan ${ }^{1}$ and Yun Li
}

\begin{abstract}
Background: Glutamate is of great importance in food and pharmaceutical industries. There is still lack of effective statistical approaches for fault diagnosis in the fermentation process of glutamate. To date, the statistical approach based on generalized additive model (GAM) and bootstrap has not been used for fault diagnosis in fermentation processes, much less the fermentation process of glutamate with small samples sets.

Results: A combined approach of GAM and bootstrap was developed for the online fault diagnosis in the fermentation process of glutamate with small sample sets. GAM was first used to model the relationship between glutamate production and different fermentation parameters using online data from four normal fermentation experiments of glutamate. The fitted GAM with fermentation time, dissolved oxygen, oxygen uptake rate and carbon dioxide evolution rate captured $99.6 \%$ variance of glutamate production during fermentation process. Bootstrap was then used to quantify the uncertainty of the estimated production of glutamate from the fitted GAM using $95 \%$ confidence interval. The proposed approach was then used for the online fault diagnosis in the abnormal fermentation processes of glutamate, and a fault was defined as the estimated production of glutamate fell outside the $95 \%$ confidence interval. The online fault diagnosis based on the proposed approach identified not only the start of the fault in the fermentation process, but also the end of the fault when the fermentation conditions were back to normal. The proposed approach only used a small sample sets from normal fermentations excitements to establish the approach, and then only required online recorded data on fermentation parameters for fault diagnosis in the fermentation process of glutamate.
\end{abstract}

Conclusions: The proposed approach based on GAM and bootstrap provides a new and effective way for the fault diagnosis in the fermentation process of glutamate with small sample sets.

Keywords: Fermentation process, Glutamate, Generalized additive model, Bootstrap, Small samples, Fault diagnosis

\section{Background}

Batch fermentation has been widely used in food, chemical and pharmaceutical industries to produce products of high value and low yield [1-4]. Online fault diagnosis of fermentation processes is of critical importance to

\footnotetext{
*Correspondence: chunbo.liu0127@gmail.com

${ }^{1}$ Key Laboratory of Advanced Process Control for Light Industry, Ministry

of Education, Jiangnan University, 1800 Lihu Avenue, Wuxi 214122,

Jiangsu, China

Full list of author information is available at the end of the article
}

ensure safe operation and stable yield of the final product. Even small faults on process parameters can decrease the quality and yield of final products. Early diagnosis of the behavior of abnormal process allows timely and corrective actions to be taken that not only can reduce the number of rejected batches, but also prevent the adverse effects on product quality and yield, and accidents $[5,6]$. Fault diagnosis approaches in batch fermentation are needed to ensure the process and associated parameters within acceptable operation conditions [1, 7-9]. The dynamic 
behavior, strong nonlinearity, batch variations and multiplicity of operation phases make the fault diagnosis of the batch fermentation process very challenging [5, 10-13].

Multivariate statistical approaches such as multi-way principal component analysis (MPCA) and multi-way partial least-squares (MPLS) have been developed for fault diagnosis in batch fermentation processes [14-16]. But, the MPCA and MPLS methods have deficiency in solving problems with non-linear features [14-17]. These methods are based on the assumptions that the entire process data come from a single operation phase and the batch wise unfolded data follow a multivariate Gaussian distribution. Other statistical methods such as Kernel function based nonlinear PCA (KPCA), artificial neural networks (ANN) and support vector machine (SVM) have also been developed for fault diagnosis in fermentation processes [17-19]. These methods have the advantage to deal with fault problems in fermentation processes with nonlinear characteristics [20-22]. However, these methods are slow in fault detection in response to fault appearance and have random criteria for fault determination, which prevent their applications in fault diagnosis in fermentation processes [17]. In addition, these methods need substantial data to construct the model with a good performance for the fault diagnosis in fermentation process $[23,24]$, which are not suitable for small sample batch processes that cannot provide substantial training data. It is essential to further develop new and effective approaches for fault diagnosis in batch fermentation process.

Generalized additive model (GAM) is a statistical model for blending properties of generalized linear models with additive models [25-28]. GAM is a flexible and effective method for investigating non-linear relationships between the response and the set of explanatory variables with less restrictions in assumptions about the data distribution [29]. The model assumes that the dependent variables are dependent on the univariate smooth terms of independent variables rather than independent variables themselves [29]. GAM has been applied to investigate trends in water quality [30,31], organic carbon content in soil [32] and factors affecting microcystin cellular quotas in the lake [29].

Bootstrap or bootstrap re-sampling was introduced as a computer-based method to calculate confidence intervals for parameters in circumstances where standard methods cannot be applied [33, 34]. It can draw a large number of re-sampled data from original data and it depends on fewer assumptions than classical statistical methods. Bootstrap can increase the robustness of fitted model in which a group of re-sampled data can be stochastically re-arranged to improve generalization capability of the fitted model [35-38]. Bootstrap methods are also an alternative for cross-validation in regression procedures when the number of observations is quite small and a validation set cannot be constructed from the original dataset $[34,39]$. Bootstrap is very useful in solving problems that are too complicated for traditional statistical analysis [34]. Bootstrap has been used in signal-processing applications such as computer-aided diagnosis in breast ultrasound [34] and signal detection [37], spectral interval selection [39], and testing fundamental hypotheses in ecology [40].

Glutamate is widely used in food and pharmaceutical industries, with the production exceeds 2.2 million tons per year [41, 42]. However, there is still lack of effective statistical approaches for fault diagnosis in batch fermentation process of glutamate. A hybrid support vector machine and fuzzy reasoning based fault diagnosis system has been developed for glutamate fermentation, but this can only cluster the faults into three categories (shortage, medium and excess) based on initial biotin content variation [17]. To date, the approach based on GAM and bootstrap has not been used for the fault diagnosis in fermentation processes, much less the fermentation process of glutamate with small samples. In previous work, we successfully applied the GAM method to optimize the fermentation process of glutamate with improved production of glutamate [43]. In this study, a combined approach of GAM and bootstrap was developed for the online fault diagnosis in the fermentation process of glutamate with small sample sets. GAM was first used to model the relationship between glutamate production and different fermentation parameters using data from normal fermentation experiments of glutamate. The fitted GAM with fermentation time (T), dissolved oxygen (DO), oxygen uptake rate (OUR) and carbon dioxide evolution rate (CER) captured $99.6 \%$ variance of glutamate production during fermentation process. Bootstrap re-sampling was then used to quantify the uncertainty of the estimated production of glutamate from the fitted GAM using $95 \%$ confidence interval. The proposed approach based on GAM and bootstrap was used for the online fault diagnosis in the abnormal fermentation processes of glutamate, and a fault was defined as the estimated production of glutamate fell outside the $95 \%$ confidence interval.

\section{Results and discussion Model construction}

The offline data on glutamate production and the online data on different fermentation parameters for model construction and validation were collected from five normal fermentation experiments of glutamate (Fig. 1). In the normal fermentation experiments, the production of glutamate increased in a non-linear way during the 

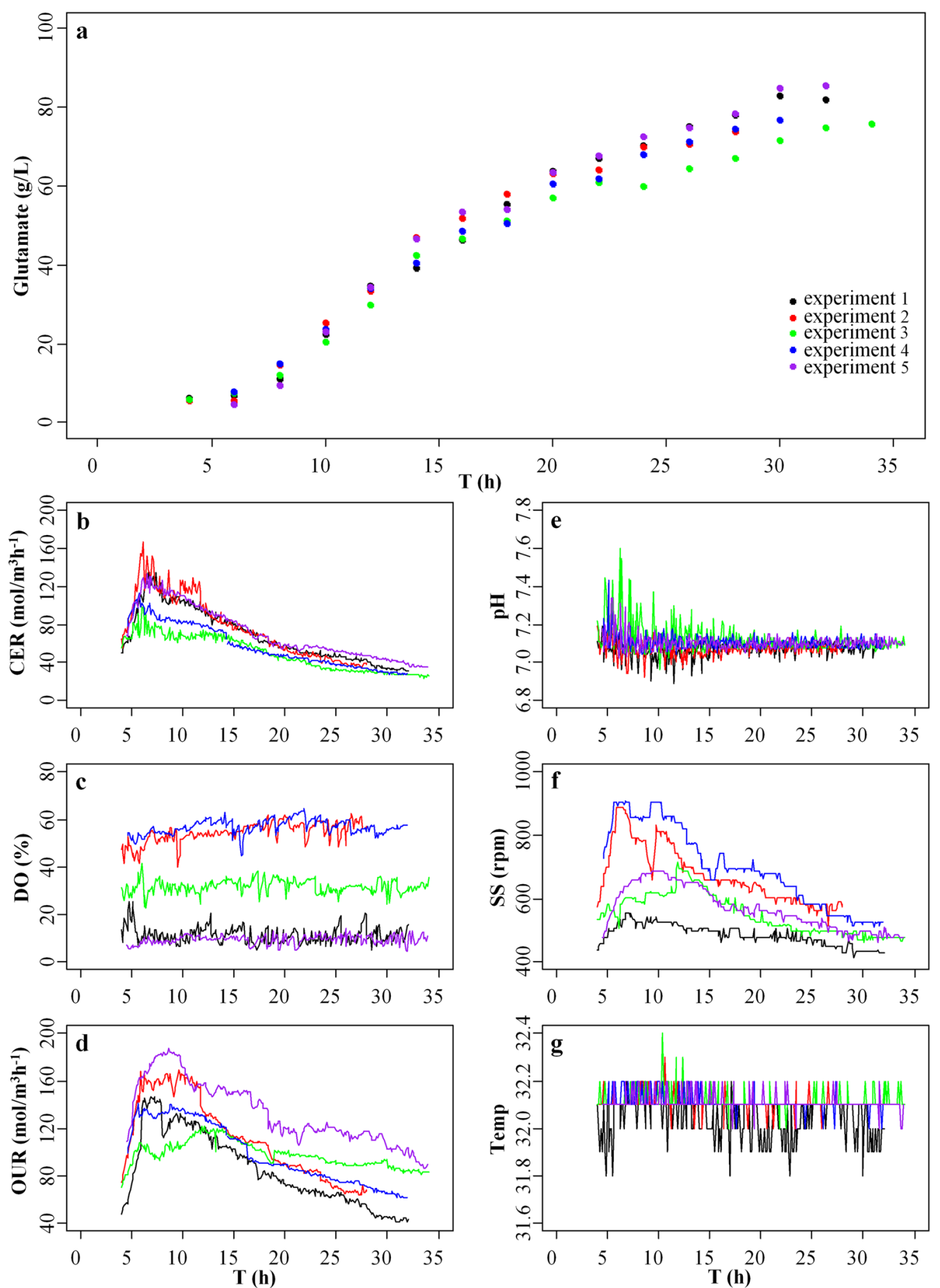

Fig. 1 Data from five normal fermentation experiments of glutamate. a the offline data on glutamate production that were measured every $2 \mathrm{~h}$ during the fermentation process; the online data on (b) carbon dioxide evolution rate (CER), c dissolved oxygen (DO), $\mathbf{d}$ oxygen uptake rate (OUR), e $\mathrm{pH}, \mathbf{f}$ stirring speed (SS) and $\mathbf{g}$ temperature (Temp) that were recorded every 6 min during the fermentation process

fermentation process with the final production of glutamate between $\sim 75$ and $\sim 85 \mathrm{~g} / \mathrm{L}$ (Fig. 1a). The levels of CER increased from $\sim 50$ to $\sim 170 \mathrm{~mol} / \mathrm{m}^{3} \mathrm{~h}^{-1}$ during the early period from 4 to $7 \mathrm{~h}$, and then dropped to $\sim 40 \mathrm{~mol} /$ $\mathrm{m}^{3} \mathrm{~h}^{-1}$ (Fig. 1b). The levels of DO of the five normal experiments were between $\sim 10$ and $\sim 55 \%$ (Fig. 1c). The 
changing trend of OUR during the formation period was similar to that of CER (Fig. 1d), which confirmed the previous observation that there was a strong link between OUR and CER during the fermentation process of glutamate [24]. The $\mathrm{pH}$ of the five normal experiments was $\sim 7.1$ (Fig. 1e), the stirring speed was between 400 and $900 \mathrm{rpm}$ (Fig. 1f), and the temperature was between 31.8 and $32.4{ }^{\circ} \mathrm{C}$ (Fig. $1 \mathrm{~g}$ ) during the fermentation period.

The training data from four randomly selected experiments were used to construct GAM and GLM. The fitted GAM showed a GCV score of 4 and an adjusted $R^{2}$ of 0.996 while the fitted GLM showed a GCV score of 44 and an adjusted $R^{2}$ of 0.940 (Table 1). This indicates that GAM was better than GLM in modeling the relationship between glutamate production and different fermentation parameters. The fitted GAM was defined as:

$$
\begin{aligned}
\text { Glutamate }= & 47.35+s(T, 7.96)+s(D O, 2.34) \\
& +s(\text { OUR }, 3.00)+s(C E R, 3.71)
\end{aligned}
$$

And, the fitted model defined by Eq. (1) can capture $99.6 \%$ variance of glutamate production. The performance of the fitted model was not significantly $(P>0.05)$ enhanced by including the remaining three fermentation parameters stirring speed, $\mathrm{pH}$ and temperature. This

Table 1 The generalized linear model and generalized additive model constructed by training data

Generalized linear model Generalized additive model

Estimates for parametric functions

$\begin{array}{lll}\text { Intercept } & 1466^{*}(573) & 47.35^{* * *}(0.22) \\ \text { T } & 2.64^{* *}(0.19) & - \\ \text { DO } & 0.02(0.08) & - \\ \text { OUR } & -0.01(0.07) & - \\ \text { CER } & -0.06^{*}(0.09) & - \\ \text { SS } & 0.01(0.02) & \\ \text { pH } & -3.01(23.69) & - \\ \text { Temp } & -45.16^{*}(17.70) & \end{array}$

Degrees of freedom for smooth terms

$\begin{array}{lll}\mathrm{s}(\mathrm{T}) & - & 7.96^{* * *} \\ \mathrm{~s}(\mathrm{DO}) & - & 2.34^{* *} \\ \mathrm{~s}(\mathrm{OUR}) & - & 3.00^{* *} \\ \mathrm{~s}(\mathrm{CER}) & - & 3.71^{* * *} \\ \text { Adjusted } & 0.940 & 0.996 \\ R^{2} & & \\ \text { GCV score } & 44 & 4\end{array}$

Data in parentheses represent standard errors of the parametric functions $T$ fermentation time, DO dissolved oxygen, OUR oxygen uptake rate, CER carbon dioxide evolution rate, SS stirring speed, Temp temperature, GCV generalized cross-validation

* $P<0.05$

** $P<0.01$

*** $P<0.001$ suggests that the production of glutamate was mainly attributed to the smooth functions of the four fermentation parameters T, DO, OUR and CER when GAM approach was used to model the relationship. And thus, the fitted GAM with the four significant factors T, DO, OUR and CER was used to estimate the production of glutamate for online fault diagnosis.

Following diagnosis was conducted to check the validity of the fitted GAM defined by Eq. (1). The sampled data and residuals generated by the fitted GAM were close to normal distribution (Fig. 2a, b), suggesting the model followed the assumption required by Eq. (2). The residuals appeared as random scatters around zero without particular trend and pattern (Fig. 2c). This indicates there were no system errors due to the fitted GAM and the capability of the model to describe the effect of different parameters on the production of glutamate. There were no obvious influential outliers between estimated and measured values of glutamate production (Fig. 2d). The performance of the fitted GAM was also confirmed by the testing data. The measured values and estimated values on glutamate production from the fitted GAM using testing data was significantly correlated $(P<0.01)$, with a correlation coefficient of 0.996 and a root mean square error of $4.16 \mathrm{~g} / \mathrm{L}$.

\section{Bootstrap re-sample and confidence interval for glutamate production}

The fitted GAM was used to estimate glutamate production during fermentation process using online recorded data of the four fermentation parameters ( $\mathrm{T}, \mathrm{CER}, \mathrm{DO}$ and OUR) from five normal fermentation experiments. The uncertainty of the estimated glutamate production was then quantified using $95 \%$ confidence interval, which were estimated from 1000 GAMs built by bootstrap resampling with replacement from the training data on glutamate production and fermentation parameters (Fig. 3). It was evident that the estimated glutamate production from the fitted GAM using online recorded data of fermentation parameters from the five normal fermentation experiments all fell within the $95 \%$ confidence interval for glutamate production; in addition, the means for glutamate production during fermentation process that were estimated from 1000 GAMs built by bootstrap resampling with replacement from the training data on glutamate production and fermentation parameters were within the estimated glutamate production from the five normal fermentation experiments. Therefore, online fault diagnosis in the fermentation process of glutamate was established by defining a fault when the estimated glutamate production from the fitted GAM fell outside the $95 \%$ confidence interval using online recorded data of the four fermentation parameters ( $\mathrm{T}, \mathrm{CER}, \mathrm{DO}$ and OUR) during the fermentation process. 


\section{Fault diagnosis during fermentation process}

Based on the $95 \%$ confidence interval for glutamate production, when there is abnormal during fermentation process, the estimated production of glutamate from the fitted GAM using online recorded data of the fermentation parameters will fall outside the $95 \%$ confidence interval, and an alarm to check the abnormal parameters can be issued immediately to avoid the decrease in the quality and production of glutamate due to fault accumulation. To demonstrate this, the fault diagnosis was conducted on two abnormal fermentation experiments of glutamate.

The fault diagnosis was firstly conducted on the abnormal fermentation experiments of glutamate with the fault source from stirring speed (Fig. 4). It was shown that the estimated glutamate production from the fitted GAM using the online recorded data of T, CER, DO and OUR from this experiment fell outside of the $95 \%$ confidence interval during the fermentation period from 12.3 to $18.5 \mathrm{~h}$ (Fig. 4a). Through the investigation on the online recorded data of different fermentation parameters, it was found that CER and OUR both fell below the level $20 \mathrm{~mol} / \mathrm{m}^{3} \mathrm{~h}^{-1}$ during the same period (Fig. $4 \mathrm{~b}, \mathrm{~d}$ ), and the level of DO was nearly close to zero (Fig. 4c). There was a sudden drop of stirring speed to below $300 \mathrm{rpm}$ during this period (Fig. 4f), and the abnormal stirring speed resulted in the very low levels of CER, DO and OUR during the same period, which could induce severe oxygen depletion to Corynebacterium Glutamicum. The actual fault in this experiment confirmed that the stirring speed of the fermenter started abnormal at about $12.3 \mathrm{~h}$, and the fault was removed at about $18.5 \mathrm{~h}$. After $18.5 \mathrm{~h}$, the levels of stirring speed, CER, DO and OUR were back to normal and the estimated glutamate production returned back to the $95 \%$ confidence interval (Fig. 4a).

The fault diagnosis was also conducted on another abnormal fermentation experiment of glutamate with the fault source from the human operation mistake that $\mathrm{NaOH}$ solution was used instead of ammonia water to maintain the $\mathrm{pH}$ level during fermentation (Fig. 5). It

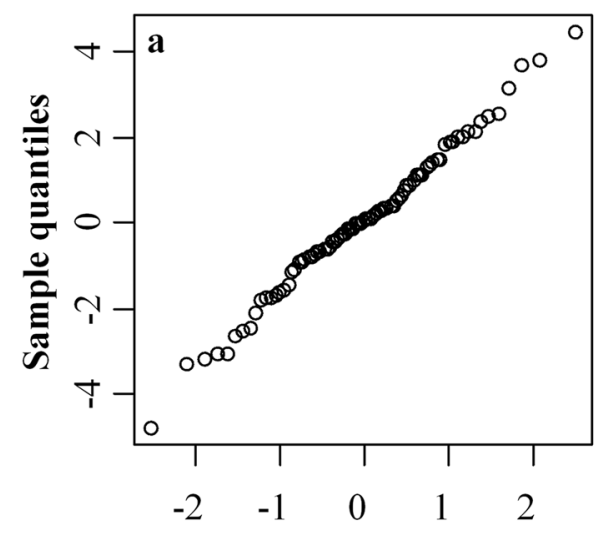

Theoretical quantiles

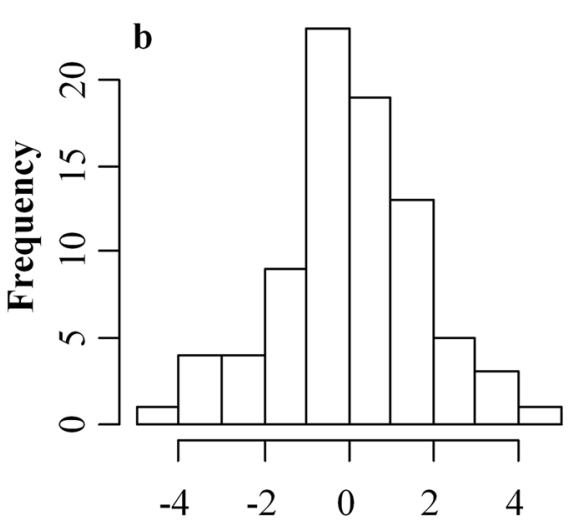

Residuals
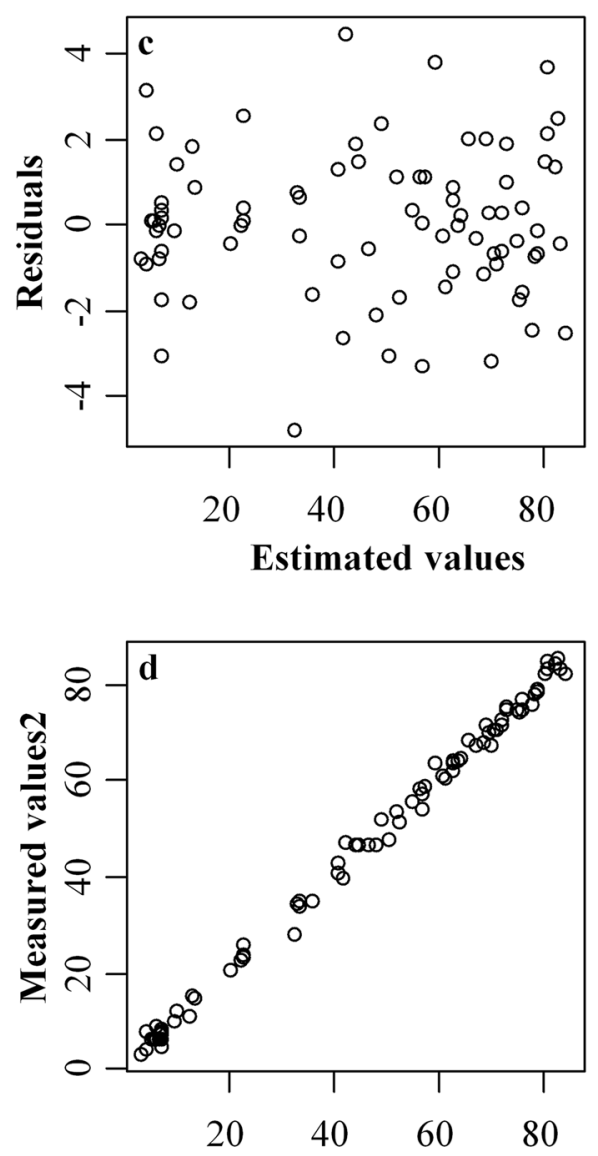

Estimated values

Fig. 2 Diagnosis of the fitted generalized additive model. a normal Q-Q plot; $\mathbf{b}$ histogram of residuals; $\mathbf{c}$ residuals versus estimated values; $\mathbf{d}$ measured versus estimated values on glutamate production 
was shown that the estimated glutamate production by the fitted GAM using the online data of T, CER, DO and OUR from this experiment fell outside the $95 \%$ confidence interval for glutamate production during the period from 13 to $20 \mathrm{~h}$ (Fig. 5a). By checking the online recoded data on different fermentation parameters, it was found that there was a drop of CER and OUR during the period from 13 to $19 \mathrm{~h}$, and a drop of stirring speed from 13 to $18 \mathrm{~h}$ while the other fermentation parameters were maintained at normal conditions (Fig. 5b-g). However, the stirring speed was within the normal range of 400-900 rpm during the period from 13 to $20 \mathrm{~h}$; this indicated that the changes of stirring speed in this experiment was not attributed to the abnormal of OUR and CER. As the stirring speed, DO and temperature were all normal in this experiment, $\mathrm{pH}$ was the parameter need to be further checked so as to find the possible fault source because the level of $\mathrm{pH}$ could be still maintained at a normal range under certain abnormal conditions. After checking, an operation mistake was found that $\mathrm{NaOH}$ solution was used instead of ammonia water to maintain the $\mathrm{pH}$ level during fermentation. Such fault was very difficult to be identified by human eyes as the level of $\mathrm{pH}$ was still maintained at a normal range when ammonia water was replaced by $\mathrm{NaOH}$ solution during the operation. But, $\mathrm{NaOH}$ solution was harmful to the growth of C. Glutamicum and it cannot serve as nitrogen source required by glutamate synthesis during the fermentation process as provided by the added ammonia water [24].

Although the fault source from the operation mistake, which $\mathrm{NaOH}$ solution was used instead of ammonia water, was not easy to be identified in this experiment by artificial check of different fermentation parameters, the abnormal condition was still detected by the proposed approach with the estimated glutamate production fell outside its $95 \%$ confidence interval. The start time of the fault was identified at $13 \mathrm{~h}$ when the estimated glutamate production fell outside the $95 \%$ confidence interval, and the end time of fault was identified at $20 \mathrm{~h}$ as after this the estimated glutamate production returned back to the $95 \%$ confidence interval (Fig. 5a). After the fault was removed at $20 \mathrm{~h}$, the final production of glutamate was $56.1 \mathrm{~g} / \mathrm{L}$ at the end of this experiment. These results suggest that if the fault source can be identified and removed timely during the fermentation process, the final production of glutamate may be still maintained at a satisfied level, although it was lower than the final production from normal experiments.

In the abnormal experiment with the fault source from stirring speed, the offline measured glutamate production showed that the fault started at $14 \mathrm{~h}$, which was about $1.7 \mathrm{~h}$ later than the fault time shown by the proposed fault diagnosis approach (Fig. 4a). In the abnormal experiment

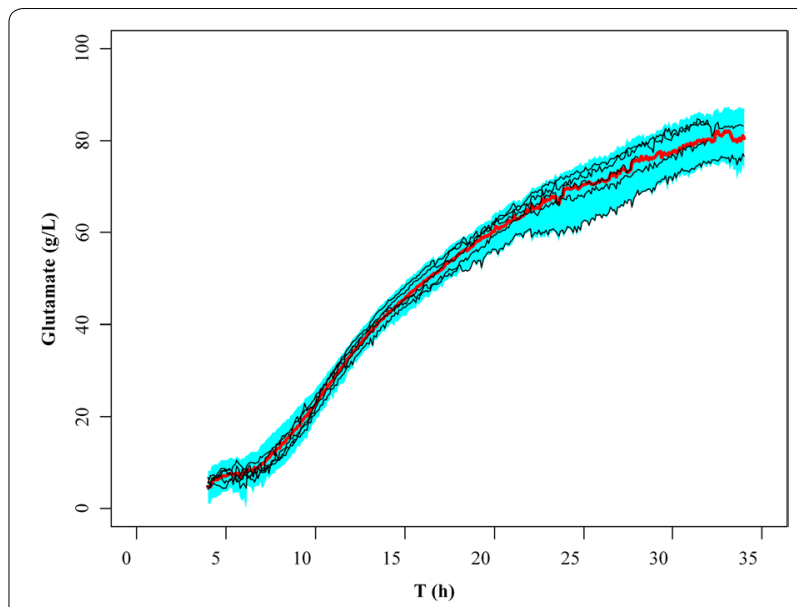

Fig. 3 The $95 \%$ confidence interval for glutamate production during fermentation process. The $95 \%$ confidence interval (shaded in green) and mean values (red curve) for glutamate production were estimated from 1000 generalized additive models (GAMs) built by bootstrap re-sampling with replacement from the training data on glutamate production and fermentation parameters. Black curves represent the estimated glutamate production for the five normal fermentation processes from the fitted GAM built by the training data using the online recorded data on fermentation parameters

with the fault source from the operation mistake that $\mathrm{NaOH}$ solution was used instead of ammonia water, the offline measured glutamate production showed that the fault started at $14 \mathrm{~h}$, which was $1 \mathrm{~h}$ later than the fault time shown by the proposed approach (Fig. 5a). Further, unlike the proposed fault diagnosis approach, the fault diagnosis based on the offline measured glutamate production cannot diagnose the end of the fault when the fault source of fermentation conditions was rectified to normal. And thus, it is noteworthy that the online fault diagnosis based on the proposed approach was very simple and effective, compared with the fault diagnosis using offline measured glutamate production. The online fault diagnosis based on the combined approach of GAM and bootstrap identified not only the start of the fault in the fermentation process, but also the end of the fault when the fermentation contentions were rectified to normal. In addition, this approach only used the online recorded data on fermentation parameters for fault diagnosis during the fermentation process, without the requirement to measure the glutamate production by taking samples.

Our approach only included the significant factors that data can also be recorded online as the parameters in the fitted model for the online fault diagnosis, rather than a model including all factors that increase the complexity of the model for online fault diagnosis. But, the faults caused by the factors that were not parameters in the fitted model can be detected timely and effectively. 

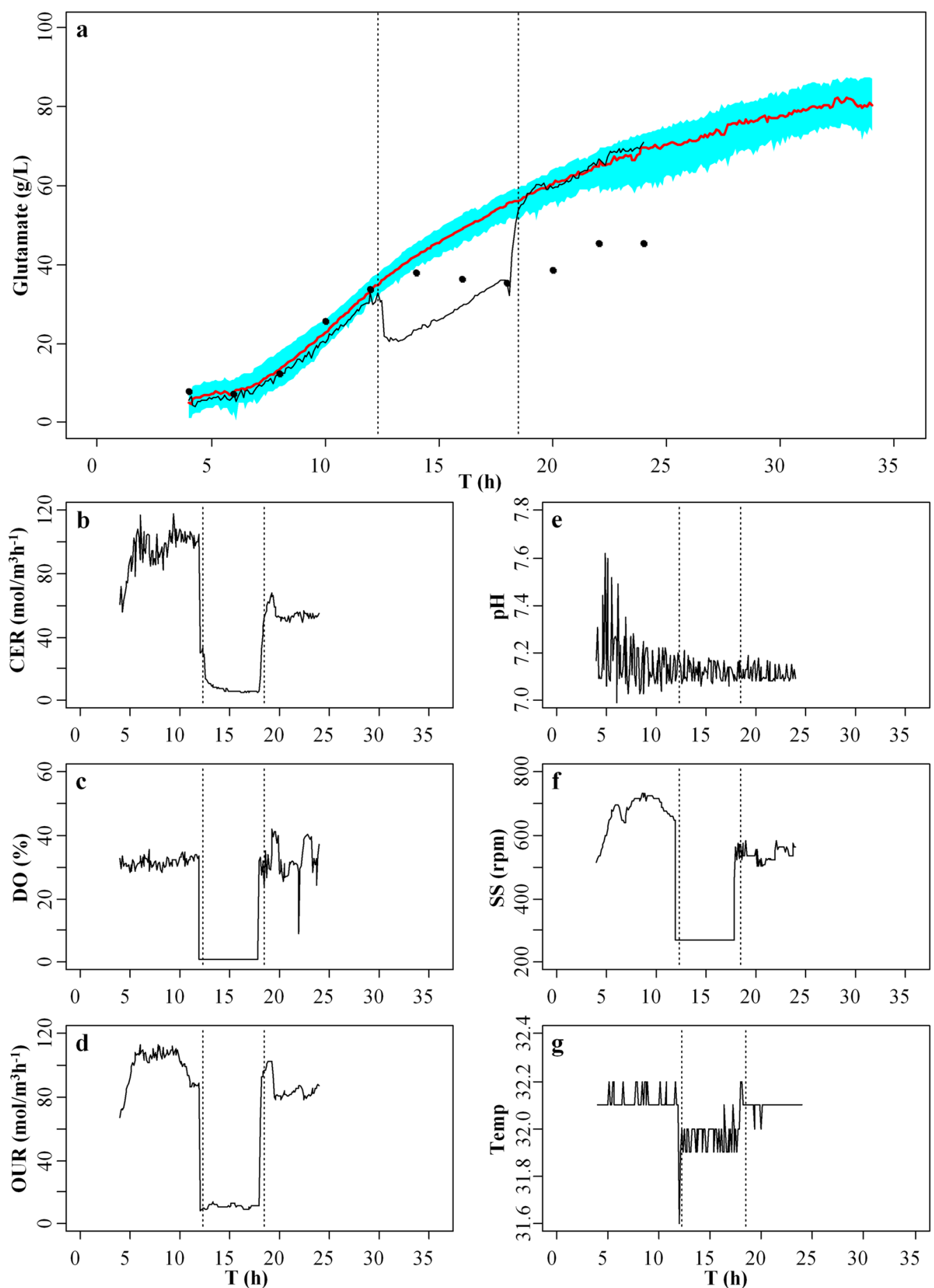

Fig. 4 Fault diagnosis in the abnormal fermentation process of glutamate with fault source from stirring speed. a the $95 \%$ confidence interval (shaded in green) and mean values (red curve) for glutamate production. The black curve represents the estimated production of glutamate from the fitted GAM using the online recorded data on fermentation parameters from this abnormal experiment. The black dots represent the offline measured production of glutamate from this abnormal experiment. b-g online recorded data on the fermentation parameter (b) carbon dioxide evolution rate (CER), c dissolved oxygen (DO), d oxygen uptake rate (OUR), e $\mathrm{pH}, \mathbf{f}$ stirring speed (SS) and $\mathbf{g}$ temperature (Temp) from this abnormal experiment 

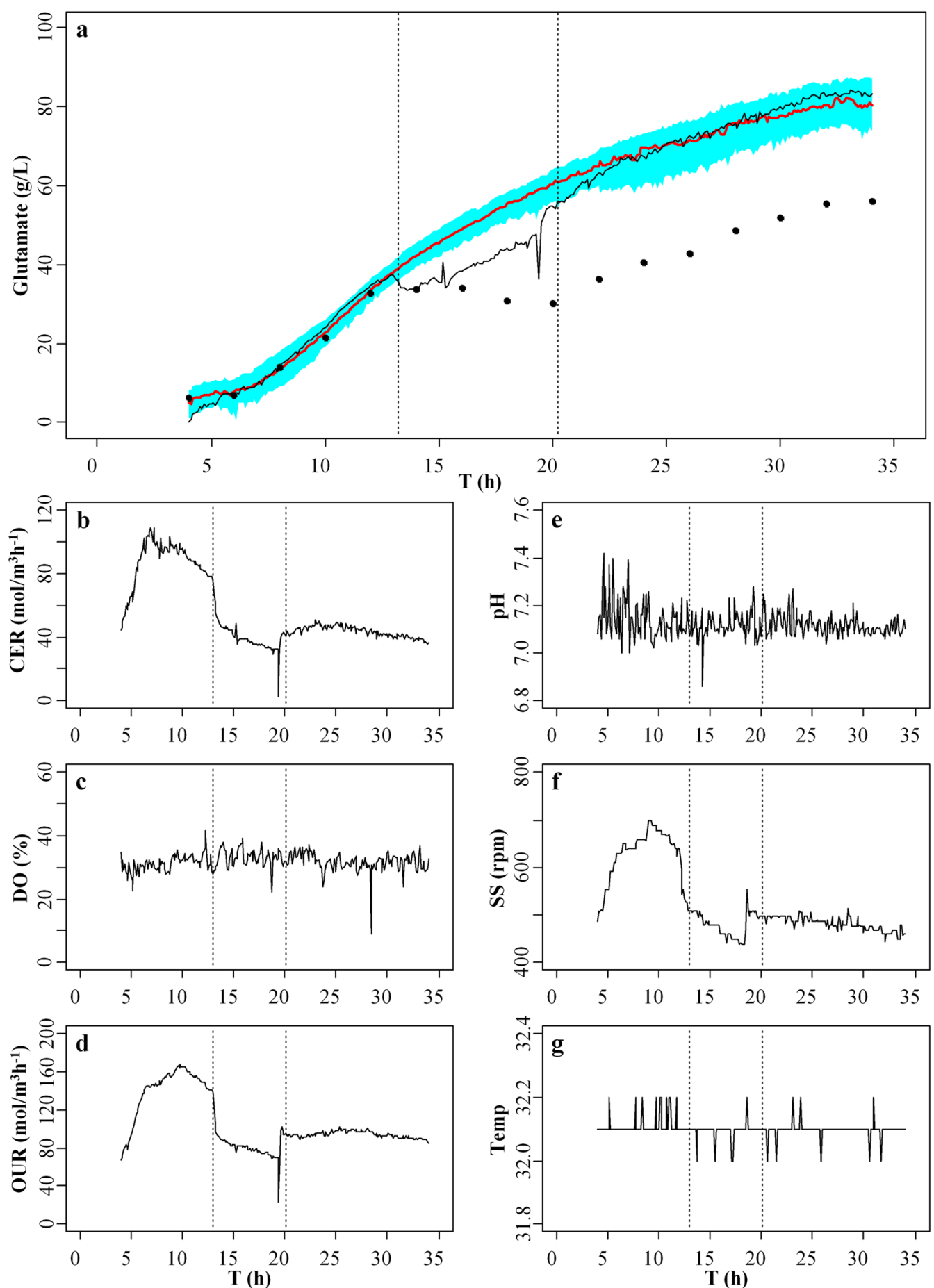

Fig. 5 Fault diagnosis in the abnormal fermentation of glutamate with fault source from the human operation mistake. The mistake was due to $\mathrm{NaOH}$ solution was used instead of ammonia water to maintain the $\mathrm{pH}$ level during the operation. a the $95 \%$ confidence interval (shaded in green) and mean values (red curve) for glutamate production. The black curve represents the estimated production of glutamate from the fitted GAM using the online recorded data on fermentation parameters from this abnormal experiment. The black dots represent the offline measured production of glutamate from this abnormal experiment. b-g online recorded data on the fermentation parameter (b) carbon dioxide evolution rate (CER), $\mathbf{c}$ dissolved oxygen (DO), $\mathbf{d}$ oxygen uptake rate (OUR), e $\mathrm{pH}$, $\mathbf{f}$ stirring speed (SS) and $\mathbf{g}$ temperature (Temp) from this abnormal experiment 
For example, for the first abnormal fermentation with fault source from stirring speed, the fault was detected effectively by the estimated glutamate production that fell outside its $95 \%$ confidence interval. In the second abnormal fermentation with the fault source from the human operation mistake, the factor $\mathrm{pH}$ was also not one of the parameters in the fitted GAM, but the fault was also detected timely and effectively by the fitted model. In addition, when $\mathrm{NaOH}$ solution was used instead of ammonia water, the level of $\mathrm{pH}$ was still maintained at a normal range during the operation mistake, but $\mathrm{NaOH}$ solution cannot serve as nitrogen source required by glutamate synthesis during the fermentation process as provided by the added ammonia water, and in this situation, the fault due to the lack of nitrogen source caused by the operation mistake was also revealed by the fitted model. These further indicate the effectiveness of the proposed approach for the online fault diagnosis in the fermentation process of glutamate.

\section{Conclusions}

This study applied the GAM and bootstrap statistical methods for the first time to the online fault diagnosis in the fermentation process of glutamate with small samples. The fitted GAM using offline measured data on glutamate production and online recorded data on different fermentation parameters captured $99.6 \%$ variance of glutamate production during fermentation process. The uncertainty of the estimated production of glutamate from the fitted GAM was quantified by bootstrap using $95 \%$ confidence interval. The $95 \%$ confidence interval for glutamate production were estimated from 1000 GAMs built by bootstrap re-sampling with replacement from the training data on glutamate production and fermentation parameters. The online fault diagnosis based on the proposed approach identified not only the start of the fault in the abnormal fermentation processes, but also the end of the fault when the fermentation conditions were back to normal. The proposed approach only need a small sample sets from normal fermentations experiments to establish the approach, and then use online recorded data on fermentation parameters for fault diagnosis in the fermentation process of glutamate, which was both time and cost-saving. Taking together, the proposed approach based on GAM and bootstrap provides a new and effective way for the online fault diagnosis in the fermentation process of glutamate with small sample sets.

\section{Methods}

\section{Microorganism}

The strain C. glutamicum S9114 used in this study was provided by the Key Laboratory of Industrial Biotechnology, Ministry of Education, Jiangnan University, China.
Seed culture was grown in sterilized liquid medium consisting of the following components (in $\mathrm{g} / \mathrm{L}$ ): $\mathrm{K}_{2} \mathrm{HPO}_{4} 1.5$, glucose $25, \mathrm{MnSO}_{4} 0.005, \mathrm{FeSO}_{4} 0.005, \mathrm{MgSO}_{4} 0.6$, corn slurry 25 and urea 2.5, with an initial pH of 7.0-7.2 on an Eberbach rotary shaker at $200 \mathrm{rpm}$ and $32^{\circ} \mathrm{C}$ for $8-10 \mathrm{~h}$.

\section{Fermentation and data collection}

The seed culture for glutamate production was then transferred into a $5 \mathrm{~L}$ fermenter (BIOTECH-5BG, Baoxing Co., China) with $3.4 \mathrm{~L}$ sterilized liquid medium consisting of the following components (in $\mathrm{g} / \mathrm{L}$ ): glucose 140, $\mathrm{K}_{2} \mathrm{HPO}_{4}$ 1.0, $\mathrm{FeSO}_{4}$ 0.002, $\mathrm{MgSO}_{4}$ 0.6, $\mathrm{MnSO}_{4}$ 0.002, thiamine $5.0 \times 10^{-5}$, corn slurry 15 and urea 3.0, with an initial $\mathrm{pH}$ of $7.0-7.2$ and at $32{ }^{\circ} \mathrm{C}$. The $\mathrm{pH}$ was maintained at $\sim 7.1$ during the fermentation process by automatically addition of $25 \%(\mathrm{w} / \mathrm{w})$ ammonia water to the liquid medium. The added ammonia water also provided the nitrogen source required by glutamate synthesis during the fermentation process [24]. DO concentrations were controlled at different levels based on experimental requirements by automatically or manually controlled agitation speed. The $\mathrm{CO}_{2}$ and $\mathrm{O}_{2}$ concentrations in the inlet and exhaust gas under the partially pressure condition were measured online by a gas analyzer (LKM2000A, Lokas Co. Ltd., Korea). Glucose was added to the fermenter according to the requirement of substrate to ensure its concentration above a suitable level $(15 \mathrm{~g} / \mathrm{L})$ during the fermentation process. The data on glutamate production were measured every $2 \mathrm{~h}$ and the data on different fermentation parameters (CER, DO, OUR, pH, SS and Temp) were online recorded every 6 min during the fermentation process. Data from five normal fermentation experiments were collected.

\section{Generalized additive model}

Generalized additive model (GAM) is the generalization of linear models that estimate the relationship between response variable and smooth functions of explanatory variables in an additive form [27, 28, 44]. As an application of GAM, considering the continuous response variable $Y$ as the production of glutamate and explanatory variables $X_{1}, \ldots, X_{p}$ as fermentation parameters (e.g., T, CER, DO, OUR, pH, SS, Temp), $Y$ is formulated as a sum of unspecified individual smooth functions of different fermentation parameters by an additive model:

$Y=c+s\left(X_{1}, m_{1}\right)+s\left(X_{2}, m_{2}\right)+\cdots+s\left(X_{p}, m_{p}\right)+\varepsilon$

where $\varepsilon$ is assumed to be normally distributed random errors with constant variance and a mean value of zero, and $s\left(X_{i}, m_{i}\right)(i=1, \ldots, p)$ are smooth functions with efficient degree of freedom $\left(m_{i} \geq 1\right)$ to be estimated from data. Generalized linear model (GLM) is a special case of GAM when $m_{i}=1$ [28]. GAM provides a useful extension of 
GLM where the smooth function $s\left(X_{i}, m_{i}\right)$ gives the ability to examine the relationship between affected factor $X_{i}$ and the predicant $Y$, despite it is linearly or non-linear related.

To establish the model for the relationship between glutamate production and different fermentation parameters, data collected from normal fermentation experiments were used for constructing GLM and GAM as defined by Eq. (2). The offline data on glutamate production measured every $2 \mathrm{~h}$ and the online data on fermentation parameters (CER, DO, OUR, $\mathrm{pH}, \mathrm{SS}$ and Temp) recorded every 6 min from five normal fermentation experiments were pooled together and then randomly separated into two groups referred to as the training data and testing data. The training data from four experiments were used to construct GLM and GAM, and the testing data from the remaining experiment were used to validate the fitted model. The best model is the one with highest value of adjusted $R^{2}$, lowest generalized cross-validation (GCV) score and least significant components that can explain the effect of different fermentation parameters on glutamate production [28]. The performance of the fitted GAM was also measured based on the correlation coefficient and root mean square error between the estimated and measured production of glutamate from the testing data. The fitted GAM was used to estimate glutamate production during fermentation process using online recorded data of fermentation parameters T, CER, DO and OUR from five normal fermentation experiments.

\section{Bootstrap re-sample and confidence interval for glutamate production}

To quantify the uncertainty of online estimated production of glutamate from the fitted GAM, a bootstrap method was then used to estimate the $95 \%$ confidence interval for glutamate production. In general, a fitted GAM based on smoothing splines to the $N$ groups sampling data $\left\{\left(X_{i}(t), Y(t)\right): i=1, \ldots, p, t=1, \ldots, N\right\}$ is

$$
\begin{aligned}
\hat{Y}(t)= & c+s\left(X_{1}(t), m_{1}\right)+s\left(X_{2}(t), m_{2}\right) \\
& +\cdots+s\left(X_{p}(t), m_{p}\right)+\varepsilon
\end{aligned}
$$

To quantify the uncertainty of glutamate production, a cumulative distribution function $G$ for the confidence interval of the prediction error $Y(N+h)-\hat{Y}(N+h)$ $(h=1, \ldots, H), \quad$ with $\quad \hat{Y}(N+h)=c+\sum_{k=1}^{P}$ $s\left(X_{k}(N+h), m_{k}\right)$ using Eq. (3), was established. A $100(1-\alpha) \%$ confidence interval for $\hat{Y}(N+h)$ based on $X_{i}(N+h)$ was given as follow:

$$
\left[\hat{Y}(N+h)+G^{-1}(\alpha / 2), \hat{Y}(N+h)+G^{-1}(1-\alpha / 2)\right]
$$

A bootstrap re-sampling approach $[45,46]$ was applied to estimate the confidence interval for $\hat{Y}(N+h)$ (glutamate production). The fitted GAM based on the training data was then used to calculate $\hat{Y}(t)$ and the residuals $e(t)=Y(t)-\hat{Y}(t)$. The error distribution $F$ was estimated by the empirical distribution of residuals that were denoted $F_{n}$, and was then used to construct bootstrapped samples by the form: $\left\{\left(X(t), Y^{*}(t)\right), t=1,2, \ldots, N\right\}$, $\left\{\left(X(N+h), Y^{*}(N+h)\right), t=1,2, \ldots, H\right\} \quad$ with $\hat{Y}^{*}(t)=\hat{Y}(t)+\varepsilon_{t}^{*}$ and $Y^{*}(N+h)=\hat{Y}(N+h)+\varepsilon_{N+h}^{*}$, where $\varepsilon_{t}^{*}$ and $\varepsilon_{N+h}^{*}$ were independently sampled from $F_{n}$; that was, they were randomly sampled with replacement from the set of residuals $\left\{e_{1}, \ldots, e_{N}\right\}$. The asterisk superscript denoted a value constructed for a particular bootstrap sample. Each bootstrapped sample was used to reconstruct GAM and get the estimated values $\hat{Y}^{*}(N+h)$, and the estimated errors $e_{N+h}^{*}=Y^{*}(N+h)-\hat{Y}^{*}(N+h)$. The empirical distribution of $e_{N+h}^{*}$, which was denoted $\tilde{G}$, was the estimated distribution of the bootstrap prediction errors, which can be used as the estimated distribution function $G$ in Eq. (4). Therefore, $100(1-\alpha) \%$ a confidence interval for can be $\hat{Y}(N+h)$ estimated as:

$$
\left[\hat{Y}(N+h)+\tilde{G}^{-1}(\alpha / 2), \hat{Y}(N+h)+\tilde{G}^{-1}(1-\alpha / 2)\right]
$$

\section{Fault diagnosis}

After obtaining the $95 \%$ confidence interval for estimated glutamate production during the fermentation process, the proposed approach based on GAM and bootstrap was used for online fault diagnosis with a fault defined as an estimated production of glutamate fell outside the $95 \%$ confidence interval. The fault diagnosis was conducted on two abnormal fermentation experiments of glutamate. The first experiment was with the fault source from abnormal stirring speed, and the other experiment was with the fault source from the human operation mistake that $\mathrm{NaOH}$ solution was used instead of ammonia water to maintain the $\mathrm{pH}$ level during the fermentation of glutamate.

\section{Authors' contributions \\ $\mathrm{CL}$ and FP conceived and designed the study. $\mathrm{CL}$ performed the experiments and statistical modelling and drafted the manuscript. CL and YL interpreted the results. FP and YL revised the manuscript. All authors read and approved the final manuscript. \\ Author details \\ ${ }^{1}$ Key Laboratory of Advanced Process Control for Light Industry, Ministry of Education, Jiangnan University, 1800 Lihu Avenue, Wuxi 214122, Jiangsu, China. ${ }^{2}$ Mathematics, Informatics and Statistics Leeuwin Centre, Common- wealth Scientific and Industrial Research Organization (CSIRO), 65 Brockway Road, Floreat, WA 6014, Australia.}

\section{Acknowledgements}

We appreciate the funding support for this research provided by the National High Technology Development 863 Program, China and CSIRO Climate Adaptation Flagship, Australia. We thank Dr Rex Lau at Mathematics, Informatics and Statistics Leeuwin Centre, CSIRO, Australia for constructive advice and discussion on this manuscript. 


\section{Competing interests}

The authors declare that they have no competing interests.

\section{Availability of data and material}

The datasets supporting the conclusions of this article are included within the article.

\section{Funding}

This work was supported by the National High Technology Development 863 Program, China and Commonwealth Scientific and Industrial Research Organization (CSIRO) Climate Adaptation Flagship, Australia.

Received: 28 April 2016 Accepted: 21 July 2016

Published online: 29 July 2016

\section{References}

1. Doan XT, Srinivasan R. Online monitoring of multi-phase batch processes using phase-based multivariate statistical process control. Comput Chem Eng. 2008;32:230-43.

2. Gunther JC, Conner JS, Seborg DE. Fault detection and diagnosis in an industrial fed-batch cell culture process. Biotechnol Progr. 2007;23:851-7.

3. Lasik M, Nowak J, Krzywonos M, Cibis E. Impact of batch, repeated-batch (with cell recycle and medium replacement) and continuous processes on the course and efficiency of aerobic thermophilic biodegradation of potato processing wastewater. Bioresour Technol. 2010;101:3444-51.

4. Sakai T, Kawashima A, Koshikawa T. Economic assessment of batch biodiesel production processes using homogeneous and heterogeneous alkali catalysts. Bioresour Technol. 2009;100:3268-76.

5. Arnold SA, Gaensakoo R, Harvey LM, McNeil B. Use of at-line and in situ near-infrared spectroscopy to monitor biomass in an industrial fed-batch Escherichia coli process. Biotechnol Bioeng. 2002;80:405-13.

6. Lim J, Lee S, Hwang S. Use of quantitative real-time PCR to monitor population dynamics of ammonia-oxidizing bacteria in batch process. J Ind Microbiol Biot. 2008;35:1339-44.

7. Camacho J, Pico J. Online monitoring of batch processes using multiphase principal component analysis. J Process Contr. 2006;16:1021-35.

8. Prasad P, Maravelias CT. Batch selection, assignment and sequencing in multi-stage multi-product processes. Comput Chem Eng. 2008;32:1106-19.

9. Wang YQ, Zhou DH, Gao FR. Iterative learning model predictive control for multi-phase batch processes. J Process Contr. 2008;18:543-57.

10. Flores-Cerrillo J, MacGregor JF. Multivariate monitoring of batch processes using batch-to-batch information. AIChE J. 2004;50:1219-28.

11. Lu N, Gao F, Yang Y, Wang F. PCA-based modeling and on-line monitoring strategy for uneven-length batch processes. Ind Eng Chem Res. 2004;43:3343-52.

12. Lu NY, Gao FR, Wang FL. Sub-PCA modeling and on-line monitoring strategy for batch processes. AIChE J. 2004;50:255-9.

13. Zhao CH, Wang FL, Lu NY, Jia MX. Stage-based soft-transition multiple PCA modeling and on-line monitoring strategy for batch processes. J Process Contr. 2007;17:728-41.

14. Kourti T. Multivariate dynamic data modeling for analysis and statistical process control of batch processes, start-ups and grade transitions. J Chemometr. 2003:17:93-109.

15. Undey C, Ertunc S, Cinar A. Online batch/fed-batch process performance monitoring, quality prediction, and variable-contribution analysis for diagnosis. Ind Eng Chem Res. 2003;42:4645-58.

16. Undey C, Cinar A. Statistical monitoring of multistage, multiphase batch processes. leee Contr Syst Mag. 2002;22:40-52.

17. Ding J, Cao Y, Mpofu E, Shi ZP. A hybrid support vector machine and fuzzy reasoning based fault diagnosis and rescue system for stable glutamate fermentation. Chem Eng Res Des. 2012;90:1197-207.

18. Kramer MA. Nonlinear principal component analysis using autoassociative neural networks. AIChE J. 1991;37:233-43.
19. Webb AR. An approach to non-linear principal components analysis using radially symmetric kernel functions. Stat Comput. 1996;6:159-68.

20. Jia MX, Xu HY, Liu XF, Wang N. The optimization of the kind and parameters of kernel function in KPCA for process monitoring. Comput Chem Eng. 2012;46:94-104.

21. Nagy ZK. Model based control of a yeast fermentation bioreactor using optimally designed artificial neural networks. Chem Eng J. 2007; 127:95-109.

22. Zhu JP, Zhou LC, Liu CB. Modeling of fermentation process based on MOACO and epsilon-SVM. Proc ICAICI. 2009;II:234-9.

23. Warnes MR, Glassey J, Montague GA, Kara B. On data-based modelling techniques for fermentation processes. Process Biochem. 1996;31:147-55.

24. Zhang CY, Shi ZP, Gao P, Duan ZY, Mao ZG. On-line prediction of products concentrations in glutamate fermentation using metabolic network model and linear programming. Biochem Eng J. 2005;25:99-108.

25. Gu C, Xiang D. Cross-validating non-Gaussian data: generalized approximate cross-validation revisited. J Comput Graph Stat. 2001;10:581-91.

26. Hastie TJ, Tibshirani RJ: Generalized additive models. CRC Press; 1990.

27. Wood SN. Fast stable direct fitting and smoothness selection for generalized additive models. J Roy Stat Soc B. 2008;70:495-518.

28. Wood S. Generalized additive models: an introduction with R. Boca Raton: CRC Press; 2006

29. Tao M, Xie P, Chen J, Qin BQ, Zhang DW, Niu Y, Zhang M, Wang Q, Wu LY. Use of a generalized additive model to investigate key abiotic factors affecting microcystin cellular quotas in heavy bloom areas of lake Taihu. Plos One. 2012;7:e32020.

30. Cox ME, Moss A, Smyth GK. Water quality condition and trend in North Queensland waterways. Mar Pollut Bull. 2005;51:89-98.

31. Morton R, Henderson BL. Estimation of nonlinear trends in water quality: an improved approach using generalized additive models. Water Resour Res. 2008:44:1-11.

32. de Brogniez D, Ballabio C, Stevens A, Jones RJA, Montanarella L, van Wesemael B. A map of the topsoil organic carbon content of Europe generated by a generalized additive model. Eur J Soil Sci. 2015;66:121-34

33. Efron B. Computers and the theory of statistics - thinking the unthinkable. SIAMR. 1979;21:460-80.

34. Chen DR, Kuo WJ, Chang RF, Moon WK, Lee CC. Use of the bootstrap technique with small training sets for computer-aided diagnosis in breast ultrasound. Ultrasound Med Biol. 2002;28:897-902.

35. Brcich RF, Zoubir AM, Pelin P. Detection of sources using bootstrap techniques. leee T Signal Process. 2002;50:206-15.

36. Brown CL, Zoubir AM. Testing for impulsive behavior: a bootstrap approach. Digit Signal Process. 2001;11:120-32.

37. Ong HT, Zoubir AM. Bootstrap-based detection of signals with unknown parameters in unspecified correlated interference. leee T Signal Process. 2003:51:135-41.

38. Zoubir AM, Iskander DR. Bootstrap methods and applications. leee Signal Proc Mag. 2007;24:10-9.

39. Bras LP, Lopes M, Ferreira AP, Menezes JC. A bootstrap-based strategy for spectral interval selection in PLS regression. J Chemometr. 2008:22:695-700

40. Heikkinen J, Makipaa R. Testing hypotheses on shape and distribution of ecological response curves. Ecol Model. 2010;221:388-99.

41. Cao Y, Mpofu E, Shi ZP. A novel metabolic model incorporating directed signal flow diagram with enzymatic activities data for evaluating glutamate yield in glutamate fermentation. Biochem Eng J. 2013;77:136-46.

42. Sano C. History of glutamate production. Am J Clin Nutr. 2009:90:728s-32s

43. Liu C, Li Y, Pan F, Shi Z. Generalised additive modelling approach to the fermentation process of glutamate. Bioresource Technol. 2011;102:4184-90.

44. Marra G, Wood SN. Coverage properties of confidence intervals for generalized additive model components. Scand J Stat. 2012:39:53-74.

45. Li Y, Smith I. A statistical downscaling model for southern Australia winter rainfall. J Climate 2009:22:1142-58.

46. Stine RA. Bootstrap prediction intervals for regression. J Am Stat Assoc. 1985:80:1026-31. 